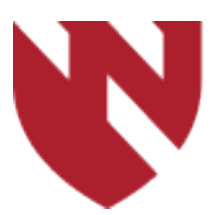

\title{
Does Prophylactic Administration of Tranexamic Acid Reduce Mean Operative Time and Perioperative Blood Loss in Posterior Cervical Spinal Fusion
}

\author{
Emmett J. Gannon \\ University of Nebraska Medical Center \\ Evan P. Larson \\ University of Nebraska Medical Center \\ Zachary C. Bailey \\ University of Nebraska Medical Center \\ Sydney Powers \\ University of Nebraska Medical Center \\ Scott A. Vincent \\ University of Nebraska Medical Center
}

Tell us how you used this information in this short survey.

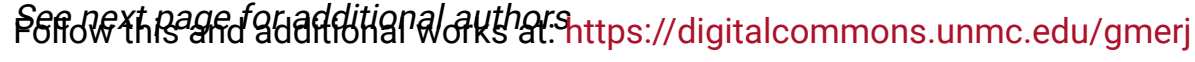

Part of the Higher Education Commons, and the Medicine and Health Sciences Commons

\section{Recommended Citation}

Gannon, E. J., Larson, E. P., Bailey, Z. C., Powers, S., Vincent, S. A., Cornett, C. A., , Lyden, L. Does

Prophylactic Administration of Tranexamic Acid Reduce Mean Operative Time and Perioperative Blood Loss in Posterior Cervical Spinal Fusion. Graduate Medical Education Research Journal. 2020 Sep 29; 2(1). https://digitalcommons.unmc.edu/gmerj/vol2/iss1/36 
Does Prophylactic Administration of Tranexamic Acid Reduce Mean Operative Time and Perioperative Blood Loss in Posterior Cervical Spinal Fusion

\section{Creative Commons License}

\section{c) (1)@ $\Theta$}

This work is licensed under a Creative Commons Attribution-Noncommercial-No Derivative Works 4.0 License.

Authors

Emmett J. Gannon, Evan P. Larson, Zachary C. Bailey, Sydney Powers, Scott A. Vincent, Chris A. Cornett, and Liz Lyden 
Conclusion: Contrary to prevailing expectations and our study hypothesis, we found that thiamine supplementation in hospitalized patients with encephalopathy did not improve LOS. Our results are consistent with the lack of beneficial effects of supplemental thiamine in other disease conditions such as sepsis (VITAMINS study) and congestive heart failure.3-5 We do not recommend routine thiamine supplementation in hospitalized patients.

\section{References}

1 Attaluri P, Castillo A, Edriss H, Nugent K. Thiamine Deficiency: An Important Consideration in Critically Ill Patients. Am J Med Sci. 2018;356(4):382-390.

2 Isenberg-Grzeda E, Kutner HE, Nicolson SE. Wernicke-Korsakoff-syndrome: under-recognized and under-treated. Psychosomatics. 2012;53(6):507-16

3 Fujii T, Luethi N, Young PJ, et al. Effect of vitamin $\mathrm{C}$, hydrocortisone, and thiamine vs hydrocortisone alone on time alive and free of vasopressor support among patients with septic shock: The VITAMINS randomized clinical trial. JAMA. 2020

https://doi.org/10.32873/unmc.dc.gmerj.2.1.033

\section{Acute Colonic Perforation in Renal Transplant Recipients: A Case Series Emily Zurbuchen ${ }^{1}$, Nathalie Sela ${ }^{2}$, Alexander Maskin ${ }^{1}$ ${ }^{1}$ University of Nebraska Medical Center, Department of Surgery \\ ${ }^{2}$ University of Nebraska Medical Center, Department of Surgery, Division of Transplantation Surgery}

Mentor: Alexander Maskin

Program: General Surgery

Type: Case Report

Introduction: There are few cases of nondiverticulitis episodes of colonic perforation following kidney transplantation throughout literature. In the systematic literature review by de'Angelis et al, emergency abdominal surgery following solid organ transplantation for non-diverticulitis causes gastrointestinal perforation accounts of $9.2 \%$ of cases, and about $58 \%$ of those perforations occurred in the colon. The aim of this case series is to review the events surrounding our two cases of colonic perforation following renal transplantation in hopes to gain understanding of this rare occurrence. Consent was obtained from the patients.

Case: Since 2011, there have been two events of transverse colonic perforation in the acute post-operative period following renal transplantation occurring at this institution. Large volume intraperitoneal air observed on plain film upon work up gastrointestinal symptoms prompted urgent surgical intervention in both cases. Both patients underwent urgent exploratory laparotomy and Hartmann procedure with resection of perforated transverse colon and proximal ostomy. The first patient underwent colostomy
4 Kattoor AJ, Goel A, Mehta JL. Thiamine therapy for heart failure: A promise or fiction? Cardiovasc Drugs Ther. 2018;32(4):313-317.

5 Smithline HA, Donnino M, Blank FSJ, et al. Supplemental thiamine for the treatment of acute heart failure syndrome: A randomized controlled trial. $B M C$ Complement Altern Med. 2019;19(1):96-019-2506-8.

\section{Proceedings of the 2nd Annual Graduate Medical Education Research Symposium | Poster Presentations}

\section{Does Prophylactic Administration of Tranexamic Acid Reduce Mean Operative Time and Perioperative Blood Loss in Posterior Cervical Spinal Fusion Surgery?}

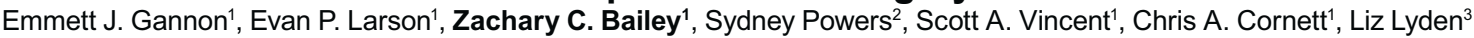

${ }^{1}$ University of Nebraska Medical Center, Department of Orthopaedic Surgery and Rehabilitation

${ }^{2}$ University of Nebraska Medical Center, College of Medicine

${ }^{3}$ University of Nebraska Medical Center, College of Public Health, Department of Biostatistics

Mentor: Chris Cornett

Program: Orthopaedic Surgery and Rehabilitation

Type: Original Research

Background: Adequate control of bleeding during spine surgery is paramount to the success and efficiency of the operation. Tranexamic acid (TXA) is a systemic antifibrinolytic that is beneficial in reducing blood loss during various orthopaedic procedures. TXA's role in posterior cervical spinal surgery, however, remains poorly described. Therefore we sought to evaluate whether prophylactic TXA administration prior to posterior cervical fusion reduces perioperative blood loss and mean operative time.

Methods: Patients undergoing three to six-level posterior cervical fusions were retrospectively enrolled into two groups. In the study group, 21 patients received TXA prior to surgery, and in the control group, 21 patients did not. Patient's younger than 19 years old, and those undergoing surgery for infection or tumors were excluded. Intraoperative blood loss, operative time, and postoperative drain output were measured in addition to demographic data.
Results: No difference existed between groups in regards to demographics. The study group had a lower mean operative time (94 min) vs the control group (110 $\mathrm{min})$, although not statistically significant $(\mathrm{p}=0.06071)$. Postoperative day 1 drain output was significantly lower in the study group $(\mathrm{p}<0.0239)$. Intraoperative blood loss, day 2, 3 and total drain output were lower in the study group but not statistically significant. There were two postoperative hematomas in the control group. No postoperative thromboembolic events occurred in either group. 
Conclusion: Perioperative TXA administration was associated with reduced mean operative time and decreased intraoperative estimated blood loss. While not statistically significant, these results may be clinically significant. Larger, higher level studies are required for further investigation.

https://doi.org/10.32873/unmc.dc.gmerj.2.1.035

\section{Does Immediate Postoperative Opioid Consumption Correlate With Long-Term Outcomes in Patients Undergoing One and Two Level Instrumented Posterior Lumbar Fusions?}

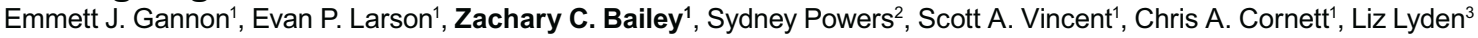

${ }^{1}$ University of Nebraska Medical Center, Department of Orthopaedic Surgery and Rehabilitation

${ }^{2}$ University of Nebraska Medical Center, College of Medicine

${ }^{3}$ University of Nebraska Medical Center, College of Public Health, Department of Biostatistics

Mentor: Chris Cornett

Program: Orthopaedic Surgery and Rehabilitation

Type: Original Research

Background: Opioid analgesics are routinely used following spine surgery. Preoperative and chronic postoperative use are associated with worse postoperative pain control and functional outcomes. Little is known about the effects of immediate postoperative use on long term outcomes. The purpose of this study is to investigate the potential relationship between increased opioid use immediately postoperatively and patient outcomes.
Methods: A retrospective review of patients undergoing instrumented 1 or 2-level posterior lumbar fusion surgery by a single surgeon was performed. Pearson correlation coefficients were used to evaluate associations between patient outcomes and immediate postoperative opioid use. Exclusion criteria included previous lumbar surgery, indications other than degenerative, and patients $<19$ years old. A total of 152 patients were included.

Results: Among 94 patients undergoing 1-level fusion, there was a significant inverse relationship between six month Short Form36 Bodily Pain and postoperative day (POD) 1,2 and total opioid use $(\mathrm{p}=0.0328, \mathrm{p}=0.0283$, and $\mathrm{p}=0.0205$ ). A significant inverse relationship was also found between 1 year Short Form-36 Physical Function and POD 1 and 2 opioid use $(\mathrm{p}=0.0415, \mathrm{p}=0.0200)$ and between 1 year Short Form-36 Bodily Pain and POD 2 opioid use ( $\mathrm{p}=0.0099)$. No correlation existed between amount of opioids consumed and outcomes after 2-level posterior lumbar fusion procedures.

Conclusion: The amount of opioid analgesics consumed in the immediate postoperative period may be helpful in predicting patient outcomes at 6 months and 1 year following 1-level posterior lumbar fusion. These findings, however, must be interpreted in context with the limitations inherent to a retrospective study.

https://doi.org/10.32873/unmc.dc.gmerj.2.1.036

\section{Rare Case of Traumatic Tricuspid Valve Injury in Patient with Sinus Venosus Atrial Septal Defect (ASD)}

Rishi Batra', Laura Newton ${ }^{1}$, Nicholas Markin², Samuel Cemaj', Zachary Bauman ${ }^{1}$

${ }^{1}$ University of Nebraska Medical Center, Department of Surgery

${ }^{2}$ University of Nebraska Medical Center, Department of Anesthesiology

Mentor: Zach Bauman

Program: General Surgery

Type: Case Report

Background: A 69-year-old male with history of congenital ASD repair as a child, atrial fibrillation and left ventricular dysfunction, presented as the restrained passenger of a motor vehicle crash. Initially complaining of left-sided chest pain, he was found to have left rib fractures 5-8 with flail physiology as well as a left tibial plateau and ulnar fracture. Due to flail chest physiology, open reduction and internal fixation (ORIF) of the ribs was scheduled.

Methods: After transthoracic echocardiogram (TTE) was completed, he was taken for ORIF. Upon intubation, he became acutely hypoxemic. Immediate left chest tube was placed, however, his clinical status remained unchanged. Emergent bronchoscopy was performed without resolution of the hypoxemia. Intraoperative transesophageal
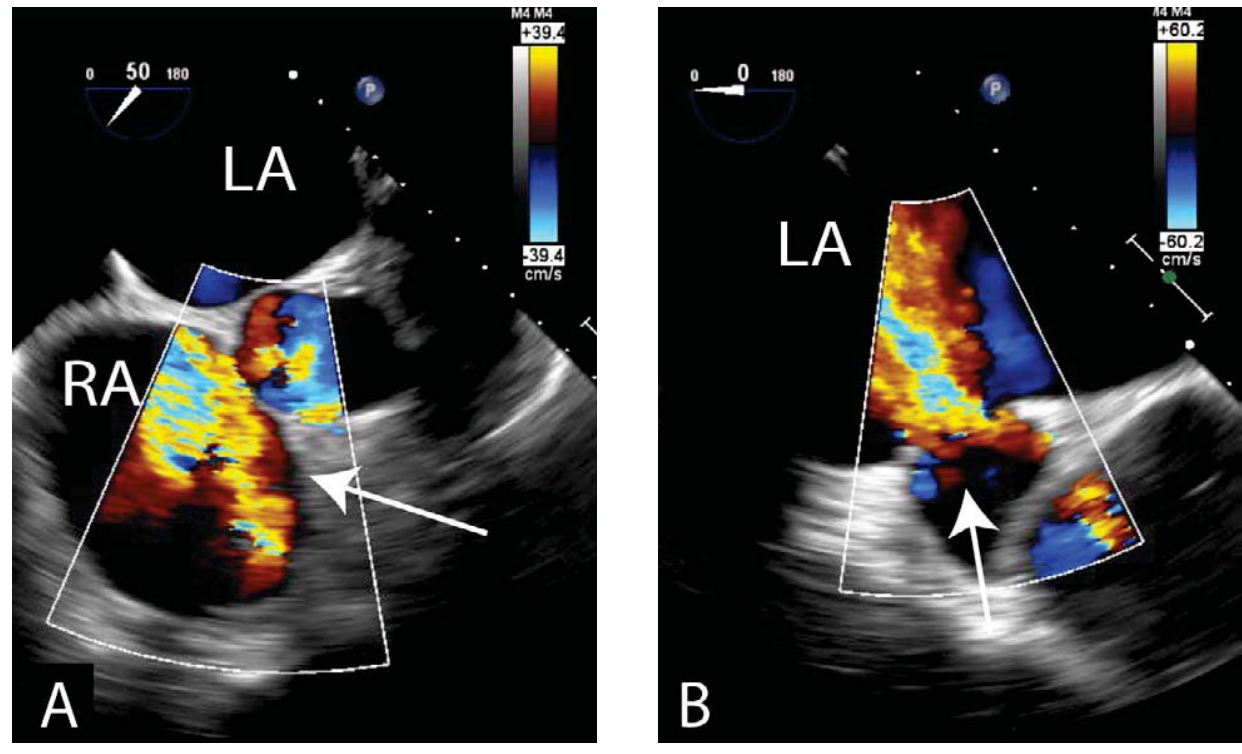

Figure 1A. Transesophageal echocardiogram showing the midesophageal modified right ventricular inflow view. There is severe tricuspid regurgitation (marked with arrow).

Figure 1B. Transesophageal echocardiogram showing a lower esophageal view of the inferior sinus venosus atrial septal defect. This image shows a high-velocity jet with right-to-left shunting. This is the result of the traumatic severe TR and the jet is directed at the sinus venosus atrial septal defect causing the right-to-left shunting (measuring $75 \mathrm{~mm} 2 \&<10 \mathrm{~mm} 2$ ). 